\title{
THEOLOGISCHE \\ GRENZÜBERSCHREITUNGEN \\ Annäherungen an einen paradoxen Begriff
}

\section{Verbot und Transgression}

Ich möchte diesen Beitrag mit einem paradoxen Zitat von G.C. Chesterton einführen: »Es gibt etwas Verdorbenes in einem Menschen, der keine Lust hat, die zehn Gebote zu brechen «. ${ }^{1}$ Das heißt, dass das Problem der Grenzüberschreitung mit dem Begriff des Gebotes bzw. des Gesetzes eng in Verbindung steht, ebenso wie es eine unauflösbare Beziehung des Gesetzes und seiner notwendigen Übertretung gibt. Die Überschreitung setzt jeweils bereits ein Verbot voraus. Deshalb konstituieren Verbot und Überschreitung einen unauflösbaren Zusammenhang, welcher im Zentrum des Denkens der französischen Philosophen Michel Foucault und George Bataille sowie der Psychoanalyse Jacques Lacans u.a. steht.

In dieser Perspektive möchte ich nun ein Gespräch zwischen einigen zeitgenössischen Stimmen der philosophischen und psychoanalytischen Kultur und der biblischen Theologie führen, um das Thema dieser Ausgabe jenseits disziplinärer Grenzziehungen zu behandeln.

»Das Verbot ist da, um verletzt zu werden «, ${ }^{2}$ schreibt George Bataille in seinem Buch über die Erotik. Und er fügt hinzu: »Diese Formulierung ist nicht, wie es zunächst scheint, eine Verstiegenheit, sondern der korrekte Wortlaut für die unvermeidliche Beziehung zwischen einander entgegengesetzten Gefühlen.« Und Michel Foucault behauptet in seinem Text A Preface to Transgression, dass Grenze und Grenzüberschreitung wesentlich aufeinander verwiesen sind. »Eine Grenze könnte nicht existieren, wenn sie absolut unüberwindbar wäre, und, umgekehrt, eine Grenzüberschreitung wäre

* Isabella Guanzini, isabella.guanzini@uni-graz.at, Institute for Fundamental Theology, Faculty of Catholic Theology, University of Graz (Austria)

1 The illustrated London news, 11. April 1925.

2 G. Bataille, Der heilige Eros, Frankfurt am M./Berlin /Wien : Ullstein 1982, 60 (»L'interdit est là pour etre violé« (G. Bataille, L'érotisme, Paris: Minuit 1957, 72). 
sinnlos, wenn sie eine bloß aus Schatten und Illusionen bestehende Grenze überwinden würde. Kann aber die Grenze ein Eigenleben jenseits des Akts, der sie glorreich überschreitet und negiert, haben? $\aleph^{3}$ George Bataille schreibt, dass es »eine tiefe Komplizenschaft zwischen Gesetz und Verstoß gegen das Gesetz« gibt, so dass beide nur in einem gegenseitigen und unüberwindbaren Spannungsverhältnis existieren können. In diesem Sinne formulieren beide Denker die tiefe Einsicht, dass nicht nur die Überschreitung unmöglich bleibt, solange noch keine Grenzen bestimmt worden sind, sondern auch, dass in die Untersagung die Notwendigkeit ihrer eigenen Überschreitbarkeit eingeschrieben ist.

Die Überschreitung ist damit eine Geste, die wesentlich mit der Grenze zu tun hat. Das Gesetz braucht das Verbrechen, um sich selbst zu behaupten, und das Verbrechen braucht ein Gesetz, um als Verbrechen verstanden werden zu können. Die jüdische Tradition sowie die mystische Lehre von Sabbatai Sevi spricht von der Beachtung religiöser Vorschriften durch ihre Überschreitung. ${ }^{4}$ In diesem Zusammenhang anerkennt der Verbrecher die Normen, die er verletzt hat: derjenige, der/die die Normen nicht kennt, kann sie auch nicht überschreiten. Die Überschreitung wird dabei von dem Begehren verursacht, das das Verbotene erregt, und von einer tiefen Angst, von einem Schuldgefühl oder Sündengefühl, wenn man es christlich interpretieren möchte, begleitet.

Bataille behauptet, dass die ersten universellen Verbote in archaischen Kulturen den Tod bzw. den Mörder und die Sexualität betroffen haben. Und er fügt hinzu: »Selbst der blutigste Mörder weiß um den Fluch, der ihn trifft. Denn der Fluch ist die Bedingung seines Ruhmes. Auch vielfache Übertretungen können dem Verbot nichts anhaben; es ist, als ob das Verbot immer nur das Mittel gewesen wäre, das, was es verwirft, mit einem ruhmvollen Fluch zu treffen «. ${ }^{5}$ Dies bedeutet, dass das Verbot nicht nur seine Beachtung verlangt: Das Umlegen einer Schranke bringt immer etwas Faszinierendes und Glorreiches mit sich. Deswegen ist es immer sehr schwierig, Gewalt einzudämmen.

Die Wahrheit des menschlichen Begehrens verbirgt sich seines Erachtens in einem gewalttätigen und dunklen Grund, welcher zwischen Verbot

3 »The limit and transgression depend on each other for whatever density of being they possess: a limit could not exist if it were absolutely uncrossable and, reciprocally, transgression would be pointless if it merely crossed a limit composed of illusions and shadows. But can the limit have a life of its own outside of the act that gloriously passes through it and negates it?" (M. Foucault, A Preface on Transgression, in Id., Language, CounterMemory, Practice: Selected Essays and Interviews, ed. by Donald F. Bouchard, New York: Cornell University Press 1980, 34).

4 Vgl. G. Scholem, Die jüdische Mystik in ihren Hauptströmungen, Frankfurt am M.: Suhrkamp 2000.

5 G. Bataille, Der heilige Eros, 44. 
und Überschreitung, Verausgabung und Begrenzung oszilliert. Überschreitung bedeutet hier allerdings keine letztliche und restlose Überwindung des Gesetzes, insofern das verbotene Objekt konstitutiv das Begehren erregt. Bataille versteht daher dieses Pulsieren von Grenzen und Grenzüberschreitung nicht als eine Dialektik, die eine höhere Synthese erreichen kann, sondern als eine Bewegung von Systole und Diastole, d.h. als eine ständige Spannung, die nicht versöhnt werden kann und die im Gegenteil beibehalten werden muss. Deshalb ist es sehr wichtig, das Gesetz aufrechtzuerhalten: Genau die ständige Spannung entspricht Bataille zufolge der Bedingung der Möglichkeit des Begehrens: Ohne Grenze entsteht keine Versuchung, ohne Gesetz verliert das Begehren seine Kraft und Vitalität. Bataille betont die Notwendigkeit dieser Spannung, um die Kraft des Begehrens, des Exzesses lebendig zu halten. In einer zu permissiven Gesellschaft würde das Begehren, ohne den Anstoß und die Erregung des Verbotes, einfach verdunsten. (Frage: Befinden wir uns nicht genau heute in einer Epoche, in welcher der Wunsch und das Begehren zunehmend verlöschen?)

Die Verbotsübertretung - schreibt Bataille - »hebt das Verbot auf, ohne es zu beseitigen. Hier verbirgt sich die treibende Kraft der Erotik, hier findet man zugleich die Triebkraft der Religionen «. ${ }^{6}$ Dieser Verweis auf die Religion ist interessant: Genau der Augenblick des Zusammentreffens von Verbot und Überschreitung, bzw. das Moment der Überschreitung selbst, entspricht Bataille zufolge der (a-moralischen und a-theologischen) mystischen Erfahrung, die nicht ohne die Negativität einer Verausgabung und eines Verlustes widerfährt. In diesem Sinne ist das Subjekt durch die Erfahrung dieser Verbindung von Gesetz und Interdikt bzw. von Gebot und Verbrechen in die Sphäre des Heiligen eingetreten.

Grundsätzlich ist das Heilige das Objekt des Verbots: Heilig ist etwas Getrenntes und Unberührbares, das außerhalb der menschlichen profanen Verfügung steht. Und dieses Verbot, das mit dem Heiligen in Verbindung steht, erscheint gleichzeitig - wie bereits Rudolf Otto auf großartige Weise erklärt hat - als fascinans und tremendum, anziehend und ungeheuer, verboten und fesselnd.

Gleichzeitig hebt Bataille die Bedeutung des Exzesses hervor, die auch ein soziopolitisches Potenzial zeigt. Unter dem Begriff der dépense, d. h. Verausgabung, versteht er den unproduktiven, exzessiven Verbrauch oder eine spezielle Art nutzloser Verschwendung, die gegen die Ausgeglichenheit von Wirtschaft und Selbsterhaltung Widerstand leisten: Luxus, Zeremonien, Kriege, Spiele, Feste, Dichtung und Sexualität stellen besondere Formen des Verlustes, d. h. der dépense dar, welche das menschliche Leben von seinen produktiven Zwecken der Vernunft und der Arbeit befreien. Die These 
ist, dass das menschliche Leben mit der Logik der Kosten-Nutzen-Analyse nicht zufrieden sein kann, sondern es dazu bereit ist, sich zu verlieren. Diese Tendenz zur zerstörerischen Dissipation und zur erotischen Behauptung des Lebens charakterisiert Bataille zufolge den Menschen, der nicht immer auf der Suche nach seiner Lust oder seinem Gleichgewicht - mit Aristoteles gesagt, nach ihrem Guten und ihrer Glückseligkeit -, sondern nach seiner Verausgabung und seinem Genießen (»jenseits des Lustprinzips«) ist. In diesem Energieüberschuss ohne Gegenleistung - wie in der überschüssigen Energie der Sonne - manifestiert sich die heilige und herrliche Lebensvitalität gegen die demütigende Hegemonie des Nutzens.

\section{Anti-Ödipus: Überschreitung gegen das Gesetz}

Ich möchte nun kurz eine zweite Perspektive auf das paradoxale Verhältnis von Gesetz bzw. Grenze und Grenzüberschreitung skizzieren, die im Zentrum der kritischen psychosozialen Analysen von Felix Guattari und Gilles Deleuze im Werk Anti-Ödipus. Kapitalismus und Schizophrenie (1972/1980) steht. Dieses Buch hat die soziopolitischen Revolten einer ganzen europäischen Generation in den 70er Jahren inspiriert und ist zu einem Manifest geworden, welches das Begehren von autoritären Begrenzungen loslöst. Insbesondere stellt die Perspektive Guattaris und Deleuzes das abendländische-bürgerliche Verständnis des Begehrens in Frage, das seine revolutionäre Kraft in den Dienst des Gesetzes und der patriarchalen Autorität gestellt hat. In diesem Sinn zielen die beiden Autoren darauf ab, die Macht des Gesetzes mittels der Dekonstruktion seines grundlegenden Symbols, nämlich das des Ödipus, außer Kraft zu setzen.

Aber was ist die Bedeutung des Ödipus?

Der freudianische Ödipus begründet ein symbolisches Interdikt des inzestuösen Genießens. Wie kann dieses Ödipus-Gesetz formuliert werden? »Kind, Du kannst nicht deine Mutter genießen, du kannst nicht zu deiner Herkunft zurückgehen«; »Mutter, du kannst dir nicht deine Frucht wieder einverleiben, du kannst nicht deine Frucht mit dir selbst vereinigen«. Dieses Verbot, dieses archaische Interdikt, ist der Ermöglichungsgrund einer sozialen Lebensgemeinschaft und somit jeglicher Kultur. Es organisiert das soziale Leben des Kindes neu: Das Kind ist nicht mehr triebhaft auf die Mutter ausgerichtet, vielmehr eröffnen sich ihm durch die Unterbrechung des Verbotes neue Lebensmöglichkeiten im sozialen und kulturellen Kontext. Das Subjekt verschiebt sein eigenes Genießen in das vielgestaltige Netz des gesellschaftlichen-symbolischen Austausches. Das Inzesttabu, das zur Entstehung der menschlichen Zivilisationen beigetragen hat, bedeutet aber nicht nur, dass das Kind die Mutter nicht genießen darf, sondern es hat auch eine breitere symbolische Bedeutung: Es geht grundsätzlich um das 
traumatische Ereignis des Gesetzes und der Begrenzung, das auch folgendermaßen übersetzt werden kann: Man kann nicht alles wissen, alles sagen, alles genießen, man kann nicht alles haben oder sein. Nach Freud ist der Vater grundsätzlich das Symbol des Gesetzes, das die Erfahrung des Unmöglichen in das Menschliche einführt. Das Gesetz führt demnach zuerst eine Unmöglichkeit ein, nämlich das Verbot des Inzestes, durch welches das soziale Leben erst ermöglicht wird. ${ }^{7}$

Der Psychoanalytiker Jacques Lacan warnt seinerseits davor, dass es nicht wünschenswert ist, zu schnell Grenzen, Gesetze und sogar ödipale Vaterfiguren zu verabschieden. Die Macht des Ödipus betrifft die Trennung des Subjekts von seinem fusionalen Genießen (ich kann alles haben, wissen, essen, sein), das von einem zerstörenden Streben nach Totalität geprägt ist.

Der freudianischen und lacanschen Herrschaft des Ödipuskomplexes, der auf die Beachtung des väterlichen Inzesttabus, d.h. auf den repressiven Kultus des Gesetzes gegründet ist, stellen Deleuze und Guattari das AntiÖdipus-Prinzip entgegen. Es handelt sich dabei um die revolutionäre Befreiung der Körper, die als »Wunschmaschinen« jenseits des Gesetzes zu verstehen sind, d.h. als produktive Fabrik des triebhaften Genießens.

Gegen jede familiäre und konformistische Reduktion des Begehrens auf das Realitätsprinzip der bürgerlichen Gesellschaft entwickeln Guattari und Deleuze eine alternative politische Theorie, in welcher alles möglich ist. Es geht um die Erfahrung eines inzestuösen Begehrens, d. h. eines unendlichen Begehrens, das keine Grenze, kein Gesetz, kein Tabu anerkennt und nach einem absoluten Besitz, nicht nur der Mutter, sondern von allem, drängt. Das Anti-Ödipus Prinzip stellt ein einseitiges Lob des revolutionären und nonkonformistischen Charakters des Begehrens als Ort der Befreiung der Subjekte und des gesellschaftlichen Systems dar. Guattari und Deleuze haben für das Genießen der Körper als einen dezentralisierten, schizophrenen und molekularen Wunsch jenseits des Gesetzes gekämpft, gegen die konservative Tendenz der starren und orthopädischen Macht und Autorität der traditionellen Institutionen (Familie, Kirche, Armee, Psychoanalyse, u.a.). In diesem Sinne haben sie ein Begehren ohne Gesetz in der Perspektive einer vitalistischen und anarchischen Naturalisierung des Menschlichen in der disziplinären Gesellschaft des XX. Jahrhunderts behauptet.

7 »Aber Freud enthüllt uns, dass der Mensch dank des Namens-des-Vaters nicht dem sexuellen Dienst an der Mutter verhaftet bleibt, dass die Aggression gegen den Vater am Anfang des Gesetzes und das Gesetz im Dienste des Begehrens steht, das über das Inzestverbot einrichtet« (J. Lacan, »Über den ,Trieb’ bei Freud und das Begehren des Psychoanalytikers, in: Id., Schriften, II und in: C. Küpke (Hg.), Trieb und Begehren, Parados Verlag, Berlin 2007, 14). 


\section{Auswirkungen}

Der italienische Psychoanalytiker Massimo Recalcati behauptet, dass wir heute in einer inzestuösen Zeit, d.h. einer Zeit des Anti-Ödipus leben, in welcher der Zwang zu genießen, zu haben, zu konsumieren das Leben beherrscht. ${ }^{8}$ Das ist die erste Auswirkung der Abschaffung des Ödipus. Die »anarchischen Wunschmaschinen«, die sich als Befreiungen von festen und despotischen Idealen der Tradition und der gesellschaftlichen Institutionen verstanden wissen wollten, wurden paradoxerweise in das Warensystem des kapitalistischen Marktes transformiert und aufgesaugt. Das Projekt des AntiÖdipus ist deshalb nicht in die befreiten, kreativen und lebendigen Ausdrücke des Begehrens in all seinen Ausformungen, sondern vielmehr in die perversen und zynischen Strömungen des heutigen neoliberalen Diskurses gemündet.

Die List der spätkapitalistischen Vernunft besteht in der Fähigkeit, systematisch die ständige Erregung und verbreitete Orientierungslosigkeit auszunutzen und diese quasi als System zu installieren. Der ethische Imperativ des permissiven Zeitgeistes, der in radikalem Gegensatz zu den repressiven Verboten der prämodernen Gesellschaft der Ordnung und der Disziplin steht, lautet »Genieße!«. Er ist dadurch charakterisiert, dass er den Exzess zur Normalität der Existenz erhoben hat. Damit nimmt das Begehren eine despotische Form an, die heute nicht mehr aushaltbar erscheint. Es geht im Genießen unter, womit paradoxerweise das Verbot des Genießens sich umkehrt in ein Gebot zu genießen. Das »In-Form-Sein « und Imperative wie »Sei, der du bist!«, »Sei glücklich!«, oder »Enjoy yourself!« erweisen sich als das zeitgenössische globale Sollen der westlichen Gesellschaften, die keinen von allen geteilten moralischen und kulturellen Horizont anerkennen können. Spontaneität, individueller Ausdruck und Selbstrealisierung sind heute Imperative in einer Gesellschaft des allumfassenden Konsums, in der das Genießen direkt der kapitalistischen Zirkularität der Waren unterstellt ist. Der Kapitalismus ist dadurch in gewisser Hinsicht kompromittierender und unterdrückender als das antike Gebot. Pasolini drückte dies so aus: Die Untertanen sind Konsumenten geworden. ${ }^{9}$ Aber dieser Produktionsapparat erzeugt und entleert gleichzeitig das Begehren, indem es jenseits der Objekte »Nichts« gibt. Die Güter sind kein Ersatz des Begehrens, sondern Phantasmen ohne Schwerkraft, die keine Erfüllung vermitteln können.

8 Vgl. Massimo Recalcati, L’uomo senza inconscio. Figure della nuova clinica psicoanalitica. Milano: Raffaello Cortina 2010; Id.; Jacques Lacan. Vol. 1. Desiderio, godimento, soggettivazione. Milano: Raffaello Cortina 2012.

9 Vgl. Pier Paolo Pasolini. Freibeuterschriften. Die Zerstörung der Kultur des Einzelnen durch die Konsumgesellschaft, Berlin-Wilmersdorf: Wagenbach 2011. 
Darüber hinaus - und das ist die zweite Auswirkung - entspricht diese psychosoziale Lage einer Zeit des chaotischen Abdriftens und der Destabilisierung, in welcher die Subjekte in autoritäre und despotische Identitäten und neue populistische Autoritäten flüchten. Die Grenze(n) und die Reibungswiderstände, die das Begehren und die Vitalität des Subjekts befördern, werden pulverisiert. Damit wird die Existenz, die in der Lage schien, sich endlich als befreit und emanzipiert zu betrachten, äußerst empfänglich für Einflüsterungen. Ein double bind zwischen der narzisstischen Suche einer absoluten und individuellen Freiheit und indirekten Formen der Kontrolle und der Manipulation zeigt sich am Ende als nicht bewältigbar für das Subjekt, welches zwischen gehetztem Genießen und dem Fehlen des Gesetzes dahingleitet. Dies führt letztlich zur Schwächung und Erodierung der Identität.

So besehen scheint also die Entfesselung der transgressiven Kraft der Triebe nicht ohne riskante Folgen zu sein. Bereits Friedrich Nietzsche hatte von den möglichen dramatischen Auswirkungen des Todes Gottes (als Überwindung aller symbolischen Ideale und Werte der abendländischen Tradition) gewarnt. "Was taten wir, als wir diese Erde von ihrer Sonne losketteten? Wohin bewegt sie sich nun? Wohin bewegen wir uns? Fort von allen Sonnen?« ${ }^{10}$ Deshalb hat er vom Bedürfnis eines Übermenschen gesprochen, der in der Lage wäre, diese Orientierungslosigkeit und diesen Nihilismus zu ertragen. Denn in der Tat kann, wie wir heute sehen und wie Nietzsche bereits ankündigte, diese Abschaffung der Grenze bzw. religiöser und transzendenter Ideale und Autoritäten neue Idole (Fanatismus, Populismus, Szientismus und jede Art des Fundamentalismus usw.) erzeugen.

Ihrerseits waren Guattari und Deleuze sich bewusst, dass dieser antiödipale Kampf gegen die hierarchische und patriarchalische Macht des Ödipus zugunsten der schizophrenen Kraft einer Lebensform, die jene Art der Grenzziehung ablehnt, nicht ohne Gefahr war. Diesbezüglich schreiben sie im Buch Tausend Plateaus: Kapitalismus und Schizophrenie, welches das Anti-Ödipus-Projekt fortsetzt: »Hier gibt es genau die vierte Gefahr: dass die Fluchtlinie die Mauer überschreitet, aus den schwarzen Löchern ausgeht, aber dass sie sich, statt mit anderen Fluchtlinien zusammenzuhängen und ihre eigene Bedeutung zu steigern, in Zerstörung, in reine und einfache Abschaffung, in Leidenschaft zur Abschaffung bekehrt «. ${ }^{11}$ In diesem Sinne kann die Fluchtlinie des entfesselten Triebes, der jede mögliche Grenze oder Mauer ablehnt, eine Todeslinie werden, die das Subjekt und die sozialen Bindungen nicht befreit, sondern vielmehr zerstört. Das inzestuöse Begeh-

10 F. Nietzsche, Die Fröhliche Wissenschaft, in: Werke, München 1954, Band 2, 126-128.

11 G. Deleuze, Dialogues, Paris: Flammarion 1977; Ebd., Tausend Plateaus: Kapitalismus und Schizophrenie, Berlin: Merve Verlag 1993. 
ren entspricht einem blinden Zwang des Lebens zur eigenen Selbstbehauptung, die in Zerstörung ausartet.

\section{Paulus und wir}

Im letzten Teil dieser Präsentation möchte ich die Frage nach Grenzen und Grenzüberschreitung im Rahmen des theologischen Diskurses untersuchen. Da nicht nur Bataille, Foucault oder Lacan, sondern schon der Apostel Paulus in seinen Briefen in großartiger Weise diesen anthropologischen und religiösen Konflikt exemplarisch zum Ausdruck gebracht hat. Lesen wir einfach die berühmte Passage aus dem 7. Kapitel des Römerbriefes:

Heißt das nun, dass das Gesetz Sünde ist? Keineswegs! Jedoch habe ich die Sünde nur durch das Gesetz erkannt. Ich hätte ja von der Begierde nichts gewusst, wenn nicht das Gesetz gesagt hätte: Du sollst nicht begehren. Die Sünde erhielt durch das Gebot den Anstoß und bewirkte in mir alle Begierde, denn ohne das Gesetz war die Sünde tot. Ich lebte einst ohne das Gesetz; aber als das Gebot kam, wurde die Sünde lebendig, ich dagegen starb und musste erfahren, dass dieses Gebot, das zum Leben führen sollte, den Tod bringt. Denn nachdem die Sünde durch das Gebot den Anstoß erhalten hatte, täuschte und tötete sie mich durch das Gebot. Das Gesetz ist heilig und das Gebot ist heilig, gerecht und gut. Ist dann etwa das Gute mein Tod geworden? Keineswegs! Sondern die Sünde verursachte, damit sie als Sünde offenbar werde, durch das Gute meinen Tod; denn durch das Gebot sollte die Sünde sich in ihrem ganzen Ausmaß als Sünde erweisen. Wir wissen, dass das Gesetz selbst vom Geist bestimmt ist; ich aber bin Fleisch, das heißt: verkauft an die Sünde. Denn ich begreife mein Handeln nicht: Ich tue nicht das, was ich will, sondern das, was ich hasse. Wenn ich aber das tue, was ich nicht will, erkenne ich an, dass das Gesetz gut ist. Denn ich tue nicht das Gute, das ich will, sondern das Böse, das ich nicht will. Wenn ich aber das tue, was ich nicht will, dann bin nicht mehr ich es, der so handelt, sondern die in mir wohnende Sünde. Ich stoße also auf das Gesetz, dass in mir das Böse vorhanden ist, obwohl ich das Gute tun will. Denn in meinem Innern freue ich mich am Gesetz Gottes, ich sehe aber ein anderes Gesetz in meinen Gliedern, das mit dem Gesetz meiner Vernunft im Streit liegt und mich gefangen hält im Gesetz der Sünde, von dem meine Glieder beherrscht werden. Ich unglücklicher Mensch! Wer wird mich aus diesem dem Tod verfallenen Leib erretten? (Röm $7,7-24)$

Dieses Kapitel ist eine der entscheidendsten Passagen des ganzen Neuen Testaments, um die Dialektik zwischen Gesetz und Sünde zu begreifen. Die Frage des Paulus ist radikal, besonders wenn man sie im Kontext des Judentums seiner Zeit liest. Der Apostel Paulus versucht im ganzen Brief, die Falle der Perversion zu vermeiden, d. h. die Falle eines Gesetzes, das seine eigene Überschreitung nährt, um sich selbst als Gesetz durchzusetzen. »Ich hätte ja von der Begierde nichts gewusst, wenn nicht das Gesetz gesagt hätte: Du sollst nicht begehren « $(7,7)$. Der Sünde kommt ein grenzenloser Charakter genau in Verbindung mit dem Gebot zu, das einen Wunsch zum Tode er- 
zeugt. Das Interdikt entfesselt seine Überschreitung und entfacht die Sünde. Das verbotene Objekt wird unmittelbar etwas Wünschenswertes.

Es ist in diesem Kontext nicht möglich, eine systematische Exegese dieses Kapitels anzubieten. Ich möchte allerdings zumindest das paulinische Bewusstsein für das perverse und tödliche Potenzial des Gesetzes hervorheben, für ein Gesetz, das gleichzeitig jedoch ebenfalls eine Gabe des Geistes bleibt. Denn er schreibt ja auch: »Heißt das nun, dass das Gesetz Sünde ist? Keineswegs!«. Paulus drückt seine Verzweiflung und Verständnislosigkeit gegenüber seinem Handeln aus, das er nicht orientieren kann. »Denn ich tue nicht das Gute, das ich will, sondern das Böse, das ich nicht will. (...) Ich unglücklicher Mensch!« $(7,24)$. Ohne Gesetz gäbe es keine Überschreitung, kein Schuldgefühl und keine Angst - aber auch kein Begehren und kein humanisiertes Leben.

Die biblische Tradition bezeugt somit ein tiefes Bewusstsein vom komplexen und dramatischen Verhältnis von Gesetz und Begehren, von Grenze und Grenzüberschreitung, das unsere (religiöse) Lebenserfahrung prägt.

Dieses Verhältnis darf aber nicht verabsolutiert werden und nicht so weit gehen, dass die Spannung von Gesetz und Begehren zum Ort des Sinnes wird. Diese Spannung darf nicht — wie bei Bataille — Ziel des Lebens werden: Obwohl eine solche Spannung die Aufgabe hat, die Kraft des Begehrens aufrechtzuerhalten - es gibt kein Begehren ohne Grenze -, ist das Subjekt nicht in der Lage, wie es Paulus auf großartige Weise zeigt, diese Situation lange zu ertragen.

Der »Heroismus der Transgression« kann keinem Ort des Heiligen bzw. der mystischen Erfahrung — wie bei Bataille — entsprechen: Die Idee, dass die Befreiung der Erotik und das Lob der depénse unmittelbar als Basis für die Emanzipierung aus der Sklaverei des Nützlichen zu verstehen sind, bleibt zutiefst fraglich. Der Apostel Paulus bezeugt, dass sowohl die Verabsolutierung des Gesetzes als auch die Verabsolutierung des Begehrens eine Form der Obsession darstellen, die das Subjekt nicht retten kann. Sowohl die Moralität als auch die Immoralität können nicht das letzte Wort über den Sinn oder den Unsinn des Menschlichen haben.

Im Rahmen dieser Dialektik, von der wir alle betroffen sind, sollte das theologische Denken bezeugen, dass — um es mit Martin Heidegger auszudrücken - nur ein Gott uns retten kann, ${ }^{12}$ der uns davor bewahrt, Grenze, Grenzüberschreitung oder den Kampf beider (d. h.: das Bewohnen den Widerspruch) zu verabsolutieren. Die biblische Theologie zeigt uns, dass das Subjekt in dieser Erfahrung dieses Widerspruches auf jeden Fall weder 
alleingelassen ist noch dem Tod ausgeliefert wird. Sie bietet aber - auch Paulus nicht - keine einfache unmittelbare Lösung an, sondern beruft den Menschen zu einer besonderen Wachsamkeit und Geistesgegenwart.

Darüber hinaus zeigt Paulus einen dritten Weg, der als gewisse Versöhnung dieser Spannung zu verstehen ist: die Liebe, bzw. die Agape. Es geht um ein Gebot (Nächstenliebe), welches allerdings weder einem mystischen Gefühl der Vereinigung (mit Gott oder mit dem anderen) noch einer moralischen Pflicht dem Anderen gegenüber entspricht. Paulus schreibt im Ersten Brief an die Korinther: »Sie erträgt alles, / glaubt alles, / hofft alles, / hält allem stand « (1Kor 13,7). Die Agape wird zur geeignetsten Haltung für ein menschliches Durchhalten der Dialektik von Grenzen und Grenzüberschreitung. Agape ist hier allerdings nicht als ein Stachel des Todes (1Kor 15,5556), sondern vielmehr als ein Dorn im Auge (Num 33,55) zu verstehen. Was bedeutet es?

Dieser Dorn lässt uns nicht in Ruhe, er treibt uns aber auch nicht zur Verzweiflung. Das Liebesgebot stellt einen Exzess dar, der alle mögliche ethnische, religiöse und soziokulturelle Grenzziehungen, ebenso wie die üblichen Bestimmungen der Liebe als philia oder eros überschreitet und die bestehenden moralischen und kultischen Vorschriften zu erfüllen vermag. Das Gebot der Agape entspricht daher fast einer beunruhigenden Unmöglichkeit (man könnte hier an die Feindesliebe denken!), an die sich kein Mensch wirklich angleichen kann, die aber die Menschen ständig beruft und herausfordert. Das Christentum ist kein Weg des »Friedens und der Sicherheit «13, sondern eine beharrliche »Arbeit der Liebe« ( 1,3): Durch diese Arbeit der Liebe werden alle Dinge und Zustände durch eine Verschiebung von ihrer Geschlossenheit befreit, sodass ihre Immanenz unterbrochen und eröffnet wird. Nur durch diese kleine Verschiebung können wir Christen in dieser Welt sein. Alle Zeichen der Sicherheit, Absicherung und Selbstabschließung, welchen auch das Gesetz entsprechen könnte, werden außer Kraft gesetzt, sodass sich das glaubende Subjekt nie zu Hause fühlen könnte. Es geht um das eschatologische Unheimliche im Leben zwischen Gegenwart und Zukunft, welche die christliche Erfahrung strukturiert und gleichzeitig erschüttert. Es geht um den tätigen Glauben der Kinder Gottes, die zwischen Widerstand und Ergebung, Grenze und Grenzüberschreitung immer wieder hoffen und kämpfen. 
Abstract

\section{THEOLOGICAL TRANSGRESSIONS}

\section{Approaches to a paradoxical Concept}

This paper examines the essential yet ambivalent role of the law, i.e. of limits and prohibitions, within the subjective experience of desire. In order to investigate the dialectics between limit and desire, it firstly focuses on the perspective of George Bataille and his analysis of eroticism. Moreover, the contribution takes into account the perspective of Gilles Deleuze and Felix Guattari, who focus on the relationship of desire to capitalist society, in order to affirm a different revolutionary economy of desire, celebrating immediate libidinal transgressions against any limitations. On the contrary, the psychoanalyst Jacques Lacan shows the absolute need of the law for the experience of desire and the process of subjectivation, since only through the Symbolic order the subject can join a sociolinguistic community. The final part of the contribution aims at enlightening possible correspondences among these perspectives, focusing on the Letters of the Apostle Paul and his profound understanding of the dialectics between law and desire. The Pauline Epistles offer a significant paradigm to understand the necessary but not sufficient role of the law in the experience of the Christian believer as well as of the subject as such.

KEY words: desire, law, Bataille, Deleuze, Guattari, St. Paul, Aedipus 
